\title{
Acolhimento com classificação de risco em serviço hospitalar de emergência: avaliação do processo de atendimento
}

\section{Hospital emergency service patient reception with triage: evaluation of the care process \\ Acogida con la clasificación de riesgo en servicio de urgencia en el hospital: evaluación del proceso de atención}

\author{
José Aparecido Bellucci Júniori; Dagmar Willamowius Vituri'; Gelena Lucinéia Gomes da Silva Versa ${ }^{\text {III; }}$ \\ Priscila Satie FuruyalV; Rafhaela Calinca VidorV; Laura Misue Matsuda
}

\begin{abstract}
RESUMO: Estudo transversal, de natureza quantitativa, desenvolvido no período de agosto a novembro de 2011, que objetivou avaliar o processo de atendimento em serviços hospitalares de emergência que têm implantadas a diretriz Acolhimento com Classificação de Risco. A amostra se constituiu de 314 trabalhadores que atuavam há pelo menos três meses, em quatro serviços de emergência. Para a coleta de dados, utilizou-se o questionário Instrumento de Avaliação do Acolhimento com Classificação de Risco. Entre os resultados, obteve-se que, $75,5 \%$ dos trabalhadores avaliaram o processo de atendimento como precário; $11,8 \%$ como satisfatório; 9,5\% como insuficiente e; 3,2\% como ótimo. Concluiu-se que os serviços investigados, necessitam de reorganização no sentido de atender os quesitos da diretriz Acolhimento com Classificação de Risco e, com isso, melhorar os seus processos de atendimento. Palavras-Chave: Serviço hospitalar de emergência; acolhimento; triagem; enfermagem.
\end{abstract}

ABSTRACT: This quantitative, cross-sectional study, conducted from August to November 2011, evaluated the care process in hospital emergency services that have introduced the Guidelines for Patient Reception with Triage. The sample comprised 314 employees in four emergency services, who had worked there for at least three months. Data were collected using the Patient Reception with Triage Evaluation Questionnaire. It was found that $75.5 \%$ of employees rated the care process as weak; $11.8 \%$, as satisfactory; $9.5 \%$, as inadequate, and $3.2 \%$, as good. It was concluded that the services studied need to be reorganized in order to meet the requirements of the Guidelines for Patient Reception with Triage, and thereby improve their care processes. Keywords: Emergency hospital service; user embracement; triage; nursing.

RESUMEN: Estudio transversal, cuantitativo, desarrollado en el período de agosto a noviembre de 2011, que tuvo como objetivo evaluar el proceso de atención en los servicios de urgencias hospitalarios que implementan la Acogida con la Clasificación de Riesgo. La muestra estuvo conformada por 314 empleados que trabajaron durante al menos tres meses en cuatro servicios de emergencia. Para recopilar los datos, se utilizó el cuestionario Instrumento de evaluación de la Acogida con la Clasificación de Riesgo. Entre los resultados, se encontró que el 75,5\% de los empleados clasificados el proceso de atención como los barrios de tugurios; $11,8 \%$ satisfactorio; baja un 9,5\% y el 3,2\% como bueno. Se concluyó que los servicios investigados deben ser reorganizado con el fin de cumplir con los requisitos de las directrices Acogida con la Clasificación de Riesgo y así mejorar sus procesos de atención. Palabras Clave: Servicio de urgencia en hospital; acogimiento; triaje; enfermería.

\section{INTRODUÇÃO}

Na área da saúde, a introdução de novas tecnologias, a ampliação da complexidade dos serviços e a crescente exigência dos usuários por atendimento de qualidade, têm contribuído para que a avaliação seja foco de interesse de gestores e estudiosos ${ }^{1}$.
De maneira geral, avaliar consiste em atribuir juízo de valor a um objeto de investigação². Na área da saúde, a avaliação é conceituada como a aferição dos resultados obtidos a partir da assistência prestada, devendo refletir a qualidade da estrutura e dos pro-

'Enfermeiro. Mestre em Enfermagem. Docente da Universidade Estadual do Norte do Paraná. Setor de Enfermagem. Bandeirantes, Paraná, Brasil. E-mail: bellucci@uenp.edu.br

"Enfermeira. Mestre em Enfermagem. Enfermeira Gerente de Riscos Hospitalares do Hospital Universitário regional do Norte do Paraná, da Universidade Estadual de Londrina. Londrina, Parná, Brasil..E-mail: dagmar@uel.br

IIIEnfermeira. Mestre em Enfermagem. Unidade de Terapia Intensiva-Adulto do Hospital Universitário do Oeste do Paraná. Cascavel, Paraná, Brasil. E-mail: gelenaenfermagem@yahoo.com.br

IVEnfermeira. Mestranda em Enfermagem. Universidade Estadual de Maringá. Departamento de Enfermagem. Maringá, Paraná, Brasil. E-mail: priscila. satiefuruya@gmail.com

vEnfermeira. Hospital Santa Rita de Maringá. Setor Enfermagem. Maringá, Paraná, Brasil. E-mail: rafhaela_calinca@hotmail.com

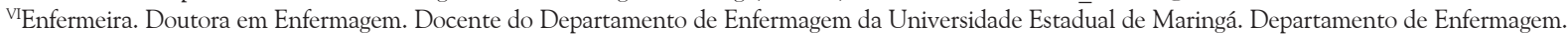

Maringá, Paraná, Brasil. E-mail: 1mmatsuda@uem.br 
cessos de atendimento ${ }^{3}$. Nesse aspecto, a avaliação, é considerada componente indispensável porque, além de fazer parte da ação de planejamento e gestão ${ }^{4}$, pode identificar fatores que interferem no atendimento.

Em se tratando de serviços hospitalares de emergência (SHE), principalmente da implantação de novos programas/diretrizes/tecnologias, a avaliação dos processos de atenção se fazem prementes ${ }^{5}$ porque se referem aos procedimentos realizados e subsidiam a tomada de decisão pelos gestores ${ }^{3}$.

$\mathrm{Na}$ atualidade, nos SHE, existem muitos fatores que intervêm na qualidade do atendimento e que são considerados de difícil resolução, tais como: superlotação; atendimento fragmentado; conflitos e assimetrias de poder; exclusão dos usuários na porta de entrada, entre outros ${ }^{6}$. Fatores dessa natureza se constituem em fortes barreiras à qualidade por requererem investimentos financeiros elevados, grande esforço dos gestores e trabalhadores e, principalmente, empenho dos usuários em compreender os processos que envolvem a rede de atenção à saúde.

Visando melhorar a qualidade do atendimento em SHE, o Ministério da Saúde lançou em 2004, a diretriz Acolhimento com Classificação de Risco (ACCR) como um dos instrumentos-chave à modificação do processo de atenção aos usuários.

A diretriz referida propõe que o usuário seja acolhido e atendido com base em critérios de avaliação de risco pré-definidos, os quais, por meio da Consulta de Enfermagem permitem a classificação do potencial de agravamento de cada caso em um sistema de cores que significam: Vermelho = Emergência; Amarelo $=$ Urgência; Verde $=$ Menor Urgência; Azul = Não Urgência ${ }^{6,7}$. Com a aplicação da Classificação de Risco, o ACCR, busca garantir aos usuários a humanização da assistência, a acessibilidade e o atendimento mais acolhedor e menos excludente.

No Brasil, estudos recentes revelam que apesar de poucos SHE terem implantado a diretriz ACCR, esta parece ser uma estratégia eficaz ao gerenciamento da qualidade nesses serviços, visto que já se tem observado resultados positivos no processo de atendimento ${ }^{8,9}$.

Considerando que o SHE se interliga com todos os setores do hospital e que o ACCR é uma das principais diretrizes à promoção da qualidade do atendimento nesse local, este estudo se justifica porque: há escassez de publicações acerca da implantação e avaliação do processo de atendimento do ACCR no Brasil e também, pela importância dos seus resultados que poderão subsidiar ações dos gestores, voltadas à sua implantação e monitoramento nas instituições de saúde.

Com base no exposto pergunta-se: Considerando a implantação da diretriz ACCR em SHE, qual é a apreciação de trabalhadores acerca do processo de atendimento? E, para responder à questão formulada, este estudo tem como objetivo avaliar o processo de atendimento em serviços hospitalares de emergência que têm implantadas a diretriz Acolhimento com Classificação de Risco.

\section{ReVisÃo DE LITERATURA}

O Ministério da Saúde propôs em 2004 a Política Nacional de Humanização da Atenção e Gestão do SUS (HumanizaSus), a qual consiste em uma política ministerial que propõe à humanização como eixo norteador das práticas de atenção e gestão em todas as instâncias do Sistema Único de Saúde (SUS) ${ }^{10}$.

No intuito de apresentar orientações específicas relacionadas ao gerenciamento e à qualidade em SHE, nos anos de 2004 e 2009, o Ministério da Saúde lançou os documentos Acolhimento com avaliação e Classificação de Risco: um paradigma ético-estético no fazer em saúde, e Acolhimento com Classificação e Avaliação de Risco ${ }^{6}$, que tiveram por objetivo principal, apresentar o acolhimento como estratégia de interferências nos processos de trabalho em saúde?

A importância do conteúdo dos documentos citados, certamente coincide com a necessidade de reorganização dos SHE brasileiros, pois, por meio das seções referidas, é possível perceber que, além de ressaltar a importância do usuário no sistema de saúde, fornece orientações sobre a organização do processo de atendimento a partir da implantação do ACCR.

\section{Metodologia}

Estudo transversal, de natureza quantitativa, desenvolvido no período de agosto a novembro de 2011, em quatro SHE, denominados: SHE I; SHE II; SHE III e SHE IV.

O SHE I é parte de um hospital de ensino, situado na região Noroeste do estado do Paraná, iniciou as suas atividades em 20 de janeiro de 1989 e implantou o ACCR no atendimento em dezembro de 2010. Atualmente atende cerca de 47.000 pacientes/ano; possui área com 31 leitos, porém acomoda em média 90 pacientes/dia; é referência no atendimento de alta complexidade ao trauma; oferece atendimento médico presencial e de retaguarda nas principais especialidades (cardiologia, neurologia, cirurgia, ortopedia, dentre outras).

OSHE II também é parte de um hospital de ensino, situado na região Norte do estado do Paraná, que iniciou as suas atividades no ano de 1971 e implantou o ACCR no atendimento no ano de 2007. Até o ano de 2007, atendia cerca de 50.000 usuários/ano No entanto, em virtude de reforma de estrutura física ocorrida em $2008 \mathrm{e}$ 2009, passou a atender em média 40.000 pacientes/ano. Dispõe atualmente de 50 leitos, é referência no Estado para o atendimento à pacientes de alta complexidade, integra o Sistema Estadual de Atendimento de Urgência e Emergência como Hospital tipo III. 
O SHE III se situa num hospital filantrópico da região Sul do estado de São Paulo, teve suas atividades iniciadas no ano de 1947 e implantou o ACCR no atendimento no ano de 2007. Na atualidade atende cerca de 100.000 pacientes/ano, possui 12 salas para atendimento; 25 leitos efetivos; é referência no atendimento ao trauma para 27 cidades da região.

O SHE IV é parte de um hospital público, situado na mesma cidade do SHE I, iniciou suas atividades no ano de 2003 e implantou o ACCR, em abril de 2008. Atende em média 5.400 pacientes/ano, possui 20 leitos efetivos, duas salas de observação e dez para procedimentos, medicações, estoques, entre outras. Funciona nas 24 horas do dia sendo referência para as Unidades Básicas de Saúde (UBS) do município.

A amostra se constituiu de 314 sujeitos e foi definida por meio de amostragem aleatória estratificada, de alocação proporcional, coletada durante os meses de agosto a novembro de 2011. Os profissionais dos SHE foram sorteados e estratificados por categoria profissional, com composição de pelo menos $60 \%$ em cada categoria. Tendo em mãos a lista dos nomes sorteados e, mediante recusa em participar do estudo ou quando o sujeito não foi localizado em três tentativas, selecionou-se o nome subsequente e, assim sucessivamente, até que se chegasse ao final da lista.

Como critérios de inclusão consideraram-se: profissionais que atuavam diretamente no SHE (enfermagem, medicina, recepção, segurança, higiene hospitalar e assistência social); tempo de atuação no SHE igual ou superior a três meses e aceite formal em participar do estudo.

A coleta de dados, se realizou no local de trabalho, utilizando-se de um questionário autoaplicável, denominado Instrumento de Avaliação do Acolhimento com Classificação de Risco1, do qual, neste estudo, serão analisadas as questões de oito a 14 , referentes à avaliação da dimensão Processo de Atendimento, quais sejam: Segurança transmitida pela equipe ao usuário (questão oito); Atendimentos não emergenciais no ACCR (questão nove); Conhecimento do protocolo de ACCR pela equipe (questão dez); Relacionamento dos gestores com a equipe (questão 11); Clareza do fluxograma de atendimento para a equipe (questão 12); Treinamento da equipe de saúde para atuar no ACCR (questão 13) e; Reavaliação dos casos em espera por atendimento (questão 14).

Para o tratamento dos dados, foram utilizadas as planilhas eletrônicas do programa Microsoft ${ }^{\circledR}$ Excel for Windows 7.0. Em seguida, realizou-se o tratamento estatístico inferencial, aplicando-se o programa Epilnfo 3.5.3. Nesse processo, foi calculada a média dos valores dos itens e a seguir, foram comparadas os resultados com os Escores para Classificação das Dimensões de Avaliação do Instrumento1 sendo: 31.5 a $35.0=$ ótimo; 26.2 a 31.4 = satisfatório; 17.5 a 26.1 = precário e; 7.0 a 17.4
= insuficiente. $\mathrm{O}$ item Atendimentos não emergenciais no ACCR, que se apresentava na forma negativa, na fase de tratamento de dados foi positivado e aqueles que foram assinalados como sem opinião ou se encontravam em branco, foram computados com o valor três (neutro).

Quanto aos aspectos éticos, o projeto que derivou este estudo foi aprovado pelo Comitê Permanente de Ética em Pesquisas Envolvendo Seres Humanos (COPEP) da Universidade Estadual de Maringá - PR, sob o Parecer no 325/2011.

\section{Resultados e Discussão}

Dentre os 314 participantes, 114 (36,3\%) eram do sexo masculino e $200(63,7 \%)$ do sexo feminino. Com relação à categoria profissional, 28 (8,9\%) eram Enfermeiros; 150 (47,8\%) Auxiliares e/ou Técnicos de Enfermagem; cinco (1,6\%) Assistentes Sociais; 59 (18,8\%) Médicos; 31 (9,8\%) Agentes Administrativos; 30 (9,6\%) Agentes Operacionais e; 11 (3,5\%) Agentes de Segurança. As áreas de atuação dos profissionais que responderam ao instrumento de avaliação foram: 277 (93,3\%) Assistencial; 3 (1,0\%) Ensino; 15 (5,1\%) Assistência e Ensino; 1 (0,3\%) Assistência e Pesquisa e $1(0,3 \%)$ Assistência, Ensino e Pesquisa.

Em relação à idade, os participantes possuíam entre 22 e 67 anos (média de 40,5 $\pm 10,5$ anos) e o tempo de atuação na instituição foi de três meses a 35 anos (média de $8.6 \pm 8.4$ anos). A média, no que se refere ao tempo de atuação nos SHE foi 7,2 \pm 7,6 anos.

Os dados de caracterização demográfica apresentados correspondem à realidade da enfermagem brasileira, em que há predomínio de profissionais do sexo feminino e, por se tratar de setor de emergência, há preponderância de profissionais mais jovens ${ }^{11}$.

O escore de predomínio da categoria de enfermagem $(56,7 \%)$, na composição da equipe dos SHE, corresponde com a literatura em geral, a qual aponta que a enfermagem exerce papel fundamental no processo assistencial desse serviço e, por isso, compõe a maior parcela do quadro de pessoal das instituições de saúde.

Os dados da avaliação dos trabalhadores acerca do Processo de Atendimento no ACCR estão relacionados na Tabela 1.

Observa-se que, independentemente do tempo de implantação da diretriz ACCR, todos os quatro SHE avaliados classificaram, em sua maioria, o processo de atendimento como precário. Esse dado é preocupante porque o resultado da avaliação do processo de atendimento denota como ocorrem as ações de dar e receber cuidado, refletindo o atendimento dispensado pelos profissionais de saúde e a maneira que os usuários procuram e utiliza o serviço ${ }^{12}$. Para minimizar o problema detectado, sugere-se a realização de avaliações frequentes e contínuas, de modo a subsidiar futuras ações de melhorias com todos os trabalhadores. 
TABELA 1: Avaliação do Processo de atendimento em SHE. Maringá - PR, 2012.

\begin{tabular}{lccccccc}
\hline \multicolumn{1}{c}{ Instituição } & N & Média & $\mathrm{DP}(*)$ & Ótimo & Satisfatório & Precário & Insuficiente \\
\hline SHE I & 94 & 21.0 & 3.4 & - & $8(8.5 \%)$ & $74(78.7 \%)$ & $12(12.8 \%)$ \\
SHE II & 122 & 21.8 & 4.3 & $5(4.1 \%)$ & $11(9.0 \%)$ & $92(75.4 \%)$ & $14(11.5 \%)$ \\
SHE III & $32 . C ̧$ & 24.8 & 4.6 & $2(6.3 \%)$ & $9(28.1 \%)$ & $21(65.6 \%)$ & - \\
SHE IV & 66 & 23.3 & 4.0 & $3(4.5 \%)$ & $9(13.6 \%)$ & $50(75.8 \%)$ & $4(6.1 \%)$ \\
TOTAL & 314 & 22.2 & 4.2 & $10(3.2 \%)$ & $37(11.8 \%)$ & $237(75.5 \%)$ & $30(9.5 \%)$ \\
\hline
\end{tabular}

(*) Desvio Padrão.

Destaca-se que a elevada média da avaliação precária $(75,5 \%)$ entre os trabalhadores dos quatro SHE pode ter sofrido influência do crescente aumento da demanda de causas externas que, somadas às dificuldades organizacionais tais como: carência qualitativa e quantitativa de recursos humanos; estrutura física inadequada; precariedade ou falta de equipamentos e de materiais ${ }^{13}$, fragilidade do sistema de referência e contra-referência8, seguramente prejudicam o processo de atendimento no ACCR.

Observa-se que a soma das porcentagens dos registros concordo e concordo totalmente $(58,97 \%$ - item oito) referente à segurança transmitida pela equipe ao usuário, reflete que a maior parte dos trabalhadores concorda que a equipe contribui para que o usuário se sinta seguro. Ver Tabela 2.

Esse resultado, possivelmente, ocorre porque, no ACCR, o usuário não deixa o serviço sem receber algum tipo de atendimento. Além disso, com a realização da escuta qualificada, um dos pilares dessa diretriz, a maior aproximação entre o usuário e a equipe de saúde tende a ser mais estreita e efetiva ${ }^{14}$.

No que se refere à Classificação dos casos não urgentes, item nove, $85,24 \%$ dos participantes assinalaram que Concordam que todos os pacientes passam pelo ACCR. Isso significa que apesar de a média da avaliação dos quatro SHE investigados os classificarem como precários, o potencial de agravamento de cada caso é previamente identificado pela equipe de saúde a todos os pacientes que procuram pelo serviço. Esse dado tem a ver com a segurança do paciente, apontada pelos trabalhadores visto que a abordagem da equipe de saúde, já no primeiro contato do paciente com o SHE, é fundamental por permitir a classificação daqueles que necessitam serem vistos primeiro e assim garantir que os casos não urgentes possam aguardar pelo atendimento com segurança $\mathrm{a}^{15}$.

Quanto ao conhecimento do protocolo do ACCR pela equipe, item dez, mais da metade (50,59\%) dos participantes discordou que os membros da equipe de saúde dos SHE avaliados, conhecem o referido documento. Esse dado é preocupante porque, o desconhecimento de alguns membros da equipe multiprofissional, que atuam nos SHE, acerca do protocolo do ACCR pode implicar o insucesso da sua implantação, pois é por meio do mesmo que as decisões são tomadas. Entre as decisões referidas, consta a determinação do atendimento, por meio de cores, que classifica a prioridade do caso ${ }^{16}$.

Em relação à Interação entre os gestores e trabalhadores no ACCR, item 11, a soma dos discordantes corresponde a 41,01\%. Essa informação mostra que apesar de o ACCR promover acolhimento e aproximação entre a equipe de saúde, quando se fala em aceitação pelos gestores de sugestões para mudança na maneira de se operacionalizar a diretriz, ainda há muito que avançar. Estudo recente apresenta o acolhimento como uma diretriz que adota uma postura de responsabilização durante as ações de atenção e de gestão, pela qual é favorecida a confiança e o compromisso entre os atores que compõe o cenário da assistência em SHE ${ }^{17}$. Nesse sentido, salienta-se que nos serviços investigados há necessidade de maior empenho dos gestores no sentido de discutirem junto à equipe de saúde, as ações e propostas de melhorias na qualidade do ACCR.

TABELA 2: Avaliação dos itens da dimensão Processo de Atendimento do ACCR, por trabalhadores de SHE. Maringá, PR, 2012.

\begin{tabular}{ccccccccccc}
\hline $\begin{array}{c}\text { Item } \\
\text { Avaliado }\end{array}$ & \multicolumn{2}{c}{$\begin{array}{c}\text { Discordo } \\
\text { Totalmente }\end{array}$} & \multicolumn{2}{c}{ Discordo } & \multicolumn{2}{c}{$\begin{array}{c}\text { Não concordo } \\
\text { nem discordo }\end{array}$} & \multicolumn{2}{c}{ Concordo } & \multicolumn{2}{c}{$\begin{array}{c}\text { Concordo } \\
\text { Totalmente }\end{array}$} \\
\cline { 2 - 11 } & $\mathrm{f}$ & $\%$ & $\mathrm{f}$ & $\%$ & $\mathrm{f}$ & $\%$ & $\mathrm{f}$ & $\%$ & $\mathrm{f}$ & $\%$ \\
\hline $\mathbf{8}$ & 18 & 5.76 & 42 & 13.46 & 68 & 21.79 & 138 & 44.23 & 46 & 14.74 \\
$\mathbf{9}$ & 7 & 2.24 & 16 & 5.12 & 23 & 7.37 & 154 & 49.35 & 112 & 35.89 \\
10 & 51 & 16.3 & 107 & 34.29 & 45 & 14.42 & 65 & 20.83 & 44 & 14.10 \\
11 & 35 & 11.21 & 93 & 29.8 & 78 & 25.0 & 84 & 26.92 & 22 & 7.05 \\
12 & 34 & 10.89 & 103 & 33.01 & 74 & 23.71 & 78 & 25 & 23 & 7.37 \\
13 & 16 & 5.12 & 83 & 26.60 & 73 & 23.39 & 108 & 34.6 & 32 & 10.25 \\
14 & 52 & 16.66 & 96 & 30.76 & 62 & 19.87 & 71 & 22.75 & 31 & 9.93 \\
\hline
\end{tabular}


Reconhecendo que a clareza do fluxograma de atendimento do ACCR é de fundamental importância para que sejam destituídos os gargalos no atendimento em SHE, também é preocupante o percentual de $43,9 \%$ de discordantes em relação ao entendimento da maneira de se operar os fluxos no ACCR (item 12). $\mathrm{Na}$ atualidade, tem se observado que nos SHE que buscam discutir com a equipe de saúde o fluxograma de atendimento, há melhorias evidentes na qualidade do gerenciamento e do cuidado ${ }^{8}$.

O ACCR, como diretriz operacional, deve utilizar da avaliação clínica para estratificar o atendimento, a partir da priorização dos casos mais graves. Não obstante, é necessário lembrar que, após a classificação do risco, o usuário ainda deve aguardar pelo atendimento médico em local específico e deve ser reavaliado periodicamente pelo enfermeiro. Nesse sentido, não era esperado neste estudo encontrar elevado percentual $(47,42 \%)$ de respostas que sinalizassem a não ocorrência da reavaliação dos usuários que aguardam pelo atendimento médico (item 14).

Estudo realizado no SHE II, em 2011, demonstrou que a implantação do ACCR trouxe melhorias na qualidade do atendimento porque, na ocasião, dentre outras mudanças ocorria a reavaliação dos usuários que aguardavam pelo atendimento médico ${ }^{18}$. Por outro lado, os dados deste estudo apontam que o padrão de qualidade atingido no momento da implantação da diretriz daquele serviço não tem se mantido, porque, grande parte dos trabalhadores da instituição referiu que a reavaliação não ocorre regularmente. Esse fato justifica e enfatiza a necessidade de treinamentos contínuos para os profissionais envolvidos no ACCR, como mostra o percentual de $44,85 \%$ da afirmativa referente ao Treinamento da Equipe de Saúde para atuar no ACCR (item 13).

\section{Conclusão}

Neste estudo a maioria dos trabalhadores avaliou o Processo de Atendimento do ACCR como precário. Os fatores que colaboram para esse resultado se relacionaram principalmente ao desconhecimento do protocolo do ACCR por todos os membros da equipe de saúde dos SHE; às dificuldades de entendimento dos fluxos no ACCR e; à não ocorrência de reavaliação dos usuários que aguardam pelo atendimento médico.

Os resultados apontam que o processo de atendimento do ACCR, nos serviços investigados, necessita de maior incremento, principalmente nas instituições públicas de ensino, que apresentaram maior número de trabalhadores insatisfeitos.

Espera-se que os resultados desta pesquisa promovam debates e melhorias, principalmente no que se refere à atuação do enfermeiro no processo de atendimento em SHE que se utilizam do ACCR, por ser ele, o profissional responsável pela classificação e reavaliação do usuário que adentra nesses serviços.

Como limitação deste estudo aponta-se o fato de que o instrumento utilizado aplica-se somente aos trabalhadores do SHE e, com isso, se exclui a avaliação do usuário. Dessa forma sugere-se a realização de futuras investigações voltadas à avaliação do processo de atendimento em SHE que se utilizam do ACCR, sob a ótica dos usuários.

\section{REFERÊNCIAS}

1.Bellucci Júnior JA. Avaliação do acolhimento com classificação de risco em serviço hospitalar de emergência [dissertação de mestrado]. Maringá (PR): Universidade Estadual de Maringá; 2011.

2.Ferreira AS. Competências gerencias para unidades básicas do Sistema Único de Saúde. Ciênc saúde coletiva. 2004; 9: 69-76.

3.Donabedian A. Basic approaches to assessment: structure, process and outcome. In: Donabedian A. Explorations in quality assessment and monitoring. Ann Harbor: Health Administration Press; 1980. p. 77-125.

4.Bosi MLM, Uchimura KY. Avaliação da qualidade ou avaliação qualitativa do cuidado em saúde? Rev Saude Publica. 2007; 41: 150-3.

5.Dubex LS, Freese E, Reis YAC. Avaliação dos serviços de urgência e emergência da rede hospitalar de referência no Nordeste brasileiro. Cad Saúde Pública. 2010; 26: 1508-18. 6.Ministério da Saúde (Br). Acolhimento e classificação de risco nos serviços de urgência. Brasília (DF): Editora MS; 2009.

7.Ministério da Saúde (Br). Secretaria Executiva Núcleo Técnico da Política Nacional de Humanização. HumanizaSUS - acolhimento com avaliação e classificação de risco: um paradigma ético-estético no fazer em saúde. Brasília (DF): Editora MS; 2004.

8.Bellucci Júnior JA, Matsuda LM. Implantação do acolhimento com classificação de risco e uso do fluxograma analisador. Texto contexto - enferm. 2012; 21: 217-25. 9.Bellucci Júnior JA, Matsuda LM. O enfermeiro no gerenciamento à qualidade em Serviço Hospitalar de Emergência: revisão integrativa da literatura. Rev Gaúcha Enferm. 2011; 32: 797-806.

10.Ministério da Saúde (Br). Política Nacional de Humanização da Atenção e Gestão em Saúde - HumanizaSUS. Brasília (DF): Ministério da Saúde; 2004.

11.Preto VA, Pedrao LJ. O estresse entre enfermeiros que atuam em unidade de terapia intensiva. Rev esc enferm USP. 2009; 43: 841-8.

12.Donabedian A. The Quality of Care - How Can it be Assessed? JAMA. 1988; 260: 1743-8.

13.Poll MA, Lunardi VL, Lunardi Filho WD. Atendimento em unidade de emergência: organização e implicações éticas. Acta Paul Enferm. 2008; 21: 509-14.

14. Nascimento ERP, Hilsendeger BR, Neth C, Belaver GM, Bertoncello KCG. Classificação de risco na emergência: avaliação da equipe de enfermagem. Rev enferm UERJ. 2011; 19: 84-8. 
15.Albino RM, Grosseman S, Riggenbach V. Classificação de risco: uma necessidade inadiável em um serviço de emergência de qualidade. Arquivos Catarinenses de Medicina. 2007; 36: 70-5.

16.Souza RS, Bastos MAR. Acolhimento com classificação de risco: o processo vivenciado por profissional enfermeiro. Rev Min Enferm. 2008; 12: 581-6.
17.Dal Pai D, Lautert L. Sofrimento no trabalho de enfermagem: reflexos do "discurso vazio" no acolhimento com classificação de risco. Esc Anna Nery. 2011; 15:524-30 18.Rossaneis MA, Haddad MCL, Borsato FG, VannuchiI MO, Sentone ADD. Caracterização do atendimento após implantação do acolhimento, avaliação e classificação de risco em hospital público. Rev Eletr Enf. 2011; 13: 648-56. 